\title{
Moving stimuli guide retrieval and (in)validation of coordination simulations
}

\author{
Magda L. Dumitru
}

Received: 7 August 2013/Accepted: 20 February 2014/Published online: 5 March 2014 (C) Marta Olivetti Belardinelli and Springer-Verlag Berlin Heidelberg 2014

\begin{abstract}
According to theories of embodied cognition, visual stimuli can either facilitate or impede the retrieval of language meaning as multimodal perceptual simulations. Here, we introduced a novel experimental paradigm to test the hypothesis that moving stimuli (i.e., motion-defined objects) facilitate coordination comprehension. Participants read coordination descriptions and saw two colored lines that matched the descriptions. Two figures then selected the lines either by moving jointly along them or by standing each on a different line. Moving selections yielded high validation scores in conjunction trials and low validation scores in disjunction trials, whereas stationary selections yielded mitigated scores. The results demonstrate that jointly moving stimuli, which are effective cues to visual grouping, help retrieve and validate conjunction simulations composed of dependent stimuli as well as retrieve and invalidate disjunction simulations composed of independent stimuli. These findings challenge accounts based on truth-condition satisfaction that stimuli properties cannot affect language comprehension and thereby reasoning.
\end{abstract}

Keywords Coordination - Conjunction - Disjunction · Reasoning $\cdot$ Simulation $\cdot$ Truth conditions $\cdot$ Motion-defined objects

\section{L. Dumitru ( $ه)$}

Department of Cognitive Science, Macquarie University, 16 University Avenue, Sydney, NSW 2109, Australia

e-mail: magda.dumitru@gmail.com

\section{Introduction}

A common assumption in the language comprehension literature is that logical expressions in natural language share core semantic content with their counterparts in formal logic. For example, the meanings of coordinators and and or are assumed to parallel their formal definitions in terms of relations between constituent parts, which are specified by truth conditions (e.g., Fillenbaum 1974; Horn 1972; Keenan and Faltz 1985). Knowing the meaning of a conjunction (the purple and the black) or of a disjunction (the purple or the black) implies knowing whether the constituent parts in each expression obtain and whether they combine according to valid truth conditions. Specifically, conjunction is valid when it describes a visual display containing both a purple object and a black object (the true-true TT condition) and invalid otherwise. Also, disjunction is valid when it describes a visual display in which one object is either purple or black (the true-false TF and the false-true FT conditions) and invalid when neither object matches the colors mentioned (the false-false FF condition). As for TT disjunctions, they are valid under the inclusive meaning and invalid under the exclusive meaning. It is further a matter of debate which meaning is the psychological default, that is, the meaning individuals first access when hearing the word or. We shall return to this issue below. Importantly, the meaning-as-truth-conditions (MTC) account sets no further cognitive or perceptual requirements on the stimuli described by coordination expressions in order for comprehenders to validate their description. In contrast, we show in the current paper that such requirements are highly relevant and set out to test the predictions of a different theoretical framework using a novel methodology, namely the embodied cognition approach using motion-defined objects (i.e., jointly moving stimuli). 
Assumptions of the meaning-as-truth-conditions (MTC) account

Language-comprehension theories inspired by the MTC account assume that stimuli perceptual properties can only affect the meaning of logical expressions insofar as the cognitive effort they require interferes with the meaning assignment process. Specifically, when selecting a meaning out of several possible for a given utterance, comprehenders will favor a more informative albeit effortful meaning over a less informative but effortless default if perceptual stimuli are easy to process, but will stay with the default if stimuli are difficult (cf. Sperber and Wilson 1986/ 1995). For example, comprehenders may start by choosing the default inclusive meaning of the disjunction word $o r$ in 'There is a blue circle or a red circle' and construct a situation in which they see a blue circle or a red circle or both (i.e., three possibilities). However, if given the time or opportunity to compare disjunction meanings, comprehenders might choose the exclusive meaning instead and construct a situation in which there is a blue circle or a red circle, but not both (i.e., two possibilities, hence lower ambiguity). Indeed, as shown in De Neys and Schaeken (2007), individuals tend to assign less informative meanings to logical expressions when visual stimuli burden their executive cognitive resources needed for understanding logical words. Specifically, their understanding of the quantifier word some in the most unambiguous way as some but not all was prevented when they attempted to concurrently perform an unrelated task (i.e., memorize complex dot patterns). A related prediction of the MTC view is that, in situations that are spared meaning-assignment dilemmas (e.g., TF and FT disjunction conditions), stimuli properties can only affect response patterns randomly if at all. The MTC view further predicts no qualitative differences in the online processing of conjunctions and disjunctions, as stimuli properties can only affect later comprehension stages (i.e., meaning assignment).

Another assumption of the MTC account is that, unless linguistic expressions have singular meanings, one of their meanings is the default. For instance, TT disjunction has two meanings, inclusive and exclusive, of which the first is generally considered to be the default. Nevertheless, results of previous experimental studies are far from being conclusive on this point. Sternberg (1979), for instance, established a marked preference for adults to interpret disjunction exclusively in a sentence-picture verification task. He secretly placed one, both, or neither of two objects in a cardboard box and offered participants clues for determining the box contents. When presented with disjunction clues pictured on poster boards and read aloud (e.g., There is a circle in the box or there is a square in the box), most adults rejected the conclusion, printed in a booklet, that the experimenter had placed both a circle and a square in the box. In an earlier study, Paris (1973) had determined precisely the reverse, namely that participants preferred to interpret disjunction inclusively, to the point of wrongly rejecting trials where only one of two pictures matched a disjunction description. Yet in a similar study, Braine and Rumain (1981) obtained equivocal results. They asked participants to judge the truth of statements such as either there is a horse or a duck in the box, while presenting them with the box contents. Slightly more adults preferred an exclusive over an inclusive interpretation and validated more trials where only one object was in the box. Taken together, these findings challenge the MTC assumption of default coordination meanings and encourage exploration of alternative accounts.

\section{Assumptions of the embodied cognition (EC) account}

The new account of coordination comprehension we examine in the present paper illustrates the embodiedcognition approach to language comprehension (Barsalou 1999; Barsalou et al. 2008). The main tenet of EC accounts is that individuals understand language by constructing multimodal perceptual simulations based on records, in long-term memory, of neural activations that arise during actual perception. Previous studies within the EC framework have tailored their methodology to investigate simulations evoked by concrete as well as by abstract language (for a review, see Glenberg 2007). For example, Zwaan et al. (2002) showed that comprehenders mentally represent object shapes when hearing sentences about them (e.g., The ranger saw the eagle in the sky) and thus respond faster when the shape of an object in the visual display matches the shape evoked in corresponding sentences (e.g., mentioning an eagle in the sky) than when it does not (e.g., mentioning an eagle in its nest). Also, as shown in Richardson et al. (2003), people consistently match certain abstract verbs (e.g., 'respect') to the image of a vertically oriented object and other abstract verbs (e.g., 'argue') to the image of a horizontally oriented object. These and other studies suggest that language offers top-down cues to sensory perception. Indeed, research using brain imaging techniques has revealed that specific areas of the (pre)motor cortex become active during language comprehension (Hauk et al. 2004; Tettamanti et al. 2005). Other studies have shown that incidental exposure to words implying vertical motion affects relevant low-level vision (e.g., Meteyard et al. 2007) and that category labels produce top-down feedback that activates visual features previously stored with the category (Lupyan 2008).

The EC-based account of coordination comprehension we present here also assumes the primacy of language in online processing of combined visual and linguistic 
information (e.g., the visual world paradigm) as well as the principle that, in turn, sensorimotor experience shapes formation and retrieval of coordination simulations. We propose that, since people are often prompted to select both items mentioned when hearing a conjunction (e.g., Have a cup of coffee and a biscuit!), they are likely to represent conjunction as a single perceptual object (cf. Link 1983) and hence simulate conjoined items as parts of the same conceptual unit. Likewise, because people are often prompted to choose between alternatives when hearing a disjunction (cf. Braine and Rumain 1981; Fillenbaum 1974; Simons 2001), they are likely to represent disjunction (e.g., Have a biscuit or a fruit!) as two independent objects. Importantly, EC accounts make no assumptions relating to default meanings but only specify the conditions that stimuli must fulfill such that comprehenders may safely recover the meanings of associated linguistic expressions. Fluctuations in validation scores reflect the goodness of fit between stimuli types as well as individuals' willingness to validate ambiguous information.

Our proposal builds on previous findings from the visual search literature detailing differential effects triggered by conjunction tasks compared with disjunction tasks. Treisman and Gelade (1980), for instance, reported that visual search for targets defined by one or more disjunctive features (e.g., blue or curved) occurs in parallel across a spatial display, whereas search for targets defined by a conjunction of features (e.g., red and vertical) requires a serial scan through items in the display. More recently, Dumitru et al. (2013) used the visual world paradigm to highlight distinct visual sampling patterns associated with conjunction expressions as well as with disjunction expressions. The authors showed that saccades to the second of two stationary targets presented together in a visual display were launched later when the second target was mentioned in disjunction sentences than in conjunction sentences. For example, when hearing Nancy examined an ant or a cloud, participants shifted their gaze from the picture of an ant to the picture of a cloud significantly later than when hearing Nancy examined an ant and a cloud. The results by Dumitru et al. (2013) align well with findings from visual processing studies that there is no cost involved in shifting attention between two object parts, but that there is a marked cost in shifting attention between two objects (Egly et al. 1994; Lamy and Egeth 2002).

Visual-search studies have also identified perceptual grouping cues that may influence the search for conjoined features (e.g., Bundesen 1990; Duncan and Humphreys 1989; Treisman and Sato 1990). Further, motion was shown to be a particularly effective cue that helps perceivers distinguish two items from each other and from a background in visual scenes and hence determine whether two items are dependent or independent (Nakayama et al. 1995; Jiang et al. 2000; Johnson and Pashler 1990; Nissen 1985; Quinlan 1998; Sagi and Julesz 1985; Simons 1996). A large body of research has confirmed that humans perceive items that move together as being more related to each other, compared with items that move independently or that are stationary.

\section{Testing the EC account of coordination}

To test the predictions of the EC account relating to the role of perceptual information in early comprehension, that is, in retrieving the meaning of conjunctions and disjunctions (i.e., the perceptual simulations they evoke), we developed an experimental paradigm including both stationary and moving stimuli. Moving stimuli were motion defined in the sense that they were objects created by gestures, hence imaginary entities. For example, to describe the path taken by a train (e.g., the engine followed by a single wagon) arriving at a railway station, we could move both hands simultaneously from left to right. In our study, dynamic trials involved two cartoon-like figures that jointly selected two visual stimuli by moving along them. Participants' task was to decide whether conjunction and disjunction descriptions of two colored lines (the TT condition) were true or false depending on whether strong grouping cues were present (i.e., figures moving jointly) or not (i.e., motionless figures). We predicted that differences in validation rates for conjunction and disjunction descriptions would be greatest when visual cues favor single grouping, which is compatible with conjunction meaning, than when visual cues are weak and allow either single or double grouping. We therefore expect high validation scores in moving TT conjunction trials but low validation scores in moving TT disjunction trials, as well as mitigated scores across stationary trials, where participants could not determine with great certainty whether two stimuli are dependent or independent. The remaining conditions (TF, FT, and FF) served as controls, but we may not rule out scoring differences between stationary and moving trials in valid conditions (TF and FT disjunction). Response times should be orthogonal to coordination type or truth conditions, but they may differ in moving trials compared with stationary trials.

\section{Methods}

Participants

Forty-four volunteering students participated in individual sessions lasting up to $30 \mathrm{~min}$ in return for course credit. 


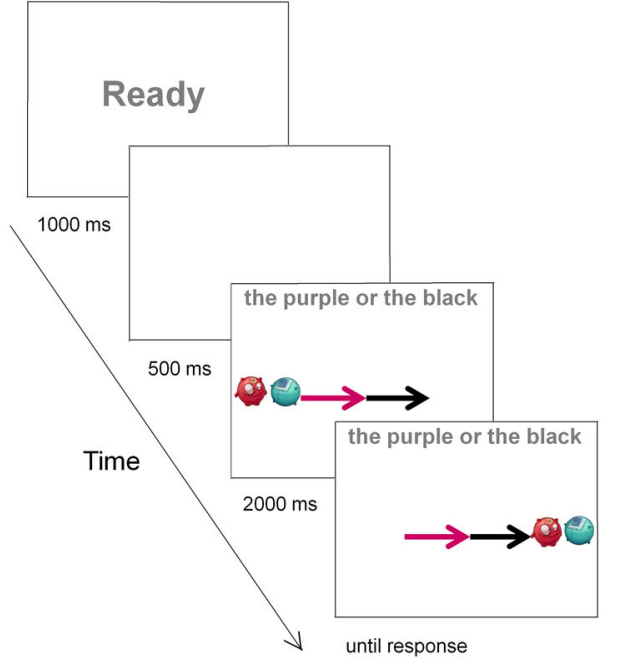

Fig. 1 Example of the sequence of events in the true-true (TT) disjunction condition. In moving trials (left-side image), the globeshaped figures moved together like a train on rails over both colored lines (here, the left line is purple, and the right line is black), thereby suggesting that lines are perceptually dependent (form a single unit). In stationary trials (right-side image), the figures stood each on a different line, thereby suggesting that lines may be perceptually

They were all native speakers of English and reported normal or corrected-to-normal vision.

\section{Stimuli}

Stimuli consisted of 96 visual displays, each containing a pair of colored lines placed horizontally next to each other (e.g., a purple line to the left and a black line to the right). Each line ended in an arrow serving as a cue to the direction in which figures could move. Line pairs were accompanied by a conjunction description (e.g., the purple and the black) or by a disjunction description (e.g., the purple or the black), as seen in Fig. 1. Line colors matched the description in all conditions except the FF condition. In half of the trials, a pair of globe-shaped figures selected one line (in the TF/FT conditions) or both lines (in the TT condition) by moving together along them like a train on rails. In the remaining trials, the figures selected one or both lines by simply standing on them.

\section{Design and procedure}

The experiment followed a 2 (Coordination: Conjunction vs. Disjunction) $\times 4$ (Condition: TT vs. TF vs. FT vs. FF) $\times 2$ (Selection: Stationary vs. Moving) factorial design. Participants were seated comfortably at approximately $40 \mathrm{~cm}$ from the computer screen and were informed that written descriptions were directions for the figures to follow and that, on each trial, they should first read the

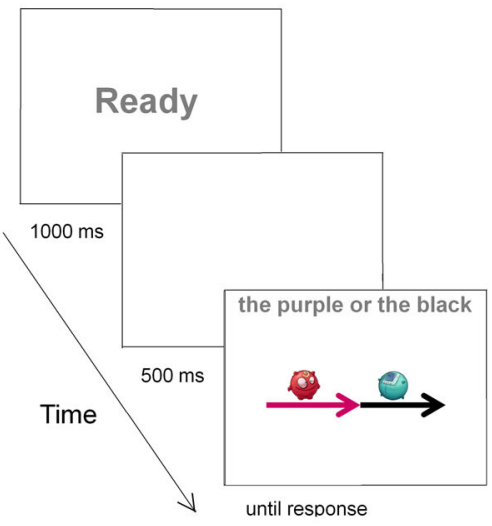

independent (form two units). In control conditions (not represented here), the figures selected one line in true-false/false-true (TF/FT) conditions and either one or both lines in false-false (FF) conditions. The colors mentioned matched line colors in all conditions except the FF condition. Conjunction trials were identical to disjunction trials save for the word 'or,' which was replaced by the word 'and' in written descriptions

description and then watch carefully how the figures select the lines. Subsequently, they should press the right button of a response box if they believed that the figures had followed the directions and the left button otherwise (counterbalanced). The structure of a trial was as follows. First, the word 'Ready' appeared in the center of the screen for 1,000 ms, followed by a 500-ms blank screen, then by a visual display featuring lines and figures. In dynamic trials, the figures started moving after 2,000 ms. Stationary displays or final displays after figures had stopped moving remained onscreen until response. The experiment consisted of 6 practice trials and 96 experimental trials presented in individually randomized orders in two equal blocks. The dependent variable was the proportion of 'correct' responses across trials; response-time data were also recorded.

\section{Results}

Response accuracy scores

Figure 2 shows the proportion of correct responses across trials. We coded correct responses as ' 1 ' and incorrect responses as '0'. By convention, 'yes' responses in disjunction TT trials were coded as 'correct,' although both 'yes' and 'no' responses are logically valid. We first conducted logistic regression analyses of results in the TT condition of interest, in order to determine whether 


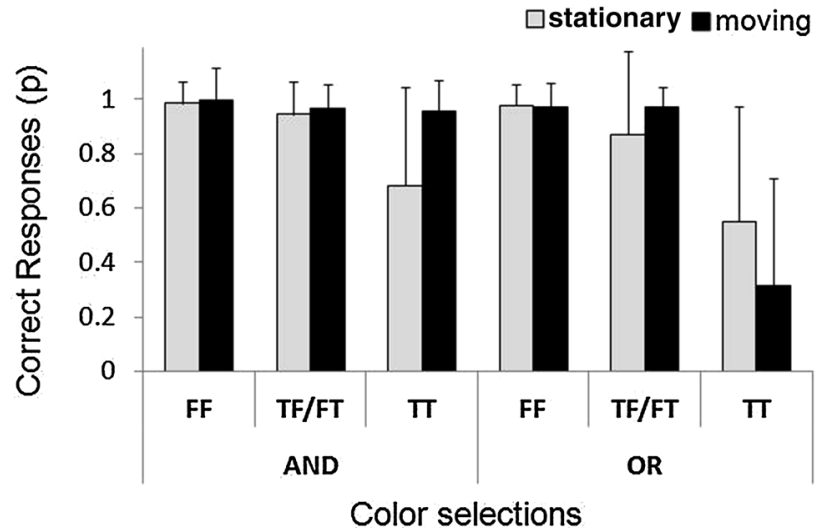

Fig. 2 Proportion of correct responses and standard deviations across true-true (TT) conditions and across control true-false/false-true (TF/ FT) and false-false (FF) conditions

selection type may be a predictor of response type ('yes' vs. 'no')

The odds ratio of validating moving versus stationary conjunction trials was 13.22 (Wald $\chi^{2}=55.19, p<.001$ ). Conversely, the odds ratio of validating stationary versus moving disjunction trials was 3.27 (Wald $\chi^{2}=40.38$, $p<.001)$. We also conducted logistic regression analyses of results in the TF/FT condition. As expected, selection type was a significant predictor of response type in disjunction trials - the odds ratio of validating moving versus stationary trials was 5.08 (Wald $\chi^{2}=40.26, p<.001$ ) —but not in conjunction trials (odds ratio of 1.64 , Wald $\chi^{2}=2.64$, $p=.104$ ). Also as expected, selection type was not a significant predictor of response type in the FF condition, neither in conjunction trials (odds ratio of validating moving vs. stationary trials was 4.04 , Wald $\chi^{2}=1.55, p=.213$ ) nor in disjunction trials (odds ratio of validating moving vs. stationary trials was .709 , Wald $\chi^{2}=.338, p=.561$ ).

Response latency scores

Figure 3 summarizes response latency scores across TT trials. A $2 \times 2$ (Selection x Connective) ANOVA revealed a main effect of selection, $F(1,43)=361.37, p<.001$, $\eta_{p}^{2}=.894(M=974 \mathrm{~ms}$ for moving trials, CI between $882 \mathrm{~ms}$ and $1,066 \mathrm{~ms}$ vs. $M=2,020 \mathrm{~ms}$ for stationary trials, CI between $1,887 \mathrm{~ms}$ and 2,154 ms), indicating that stationary stimuli are difficult to process, and an interaction between factors, $F(1,43)=17.32, p<.001, \eta_{p}^{2}=.287$, which was driven by the slightly larger differences between disjunction and conjunction processing in moving trials, $F(1,43)=26.72, p<.001, \eta_{p}^{2}=.383(M=859 \mathrm{~ms}, \mathrm{CI}$ between 774 and $944 \mathrm{~ms}$ vs. $M=1,090 \mathrm{~ms}$, CI between 972 and 1,207 ms) compared with stationary trials $F$ (1,

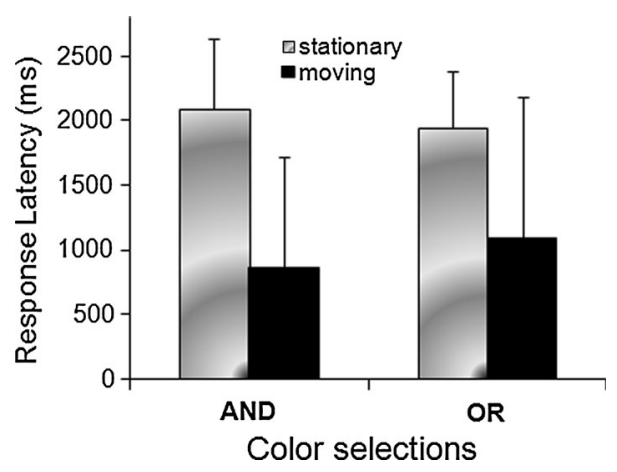

Fig. 3 Response latencies and standard deviations for true-true (TT) conditions

43) $=361.37, p=.034, \eta_{p}^{2}=.100(M=2,095 \mathrm{~ms}, \mathrm{CI}$ between 1,929 and 2,260 vs. $1,946 \mathrm{~ms}$, CI between 1,813 and $2,079 \mathrm{~ms}$ ).

\section{Discussion}

We investigated whether moving stimuli facilitate coordination comprehension and reasoning in a study inspired by EC accounts using a novel experimental paradigm (i.e., motion-defined objects). The experimental results confirmed our predictions that mitigated scores should obtain in stationary TT trials, as these are contexts where comprehenders cannot determine whether visual stimuli are dependent (form a single perceptual unit) or independent (form two perceptual units) and hence whether they correspond to the simulations evoked by conjunctions (i.e., a single object) or by disjunctions (i.e., a double object). Results also confirmed our predictions that jointly moving stimuli should elicit high validation scores in TT conjunction trials and low validation scores in TT disjunction trials, as these are contexts where comprehenders can easily determine that stimuli are dependent. Our findings suggest that experimental designs with moving stimuli can be successfully used to predict coordination validation rates, as motion is a reliable cue to inter-stimulus dependency. Dependency status is a variable that was not properly controlled for or that was overlooked in previous work. Indeed, coordination studies have used exclusively static contexts (e.g., symbols written in booklets, objects placed in boxes, or drawings on slides), where dependency status could not be determined.

The question that arises at this point is whether methodological flaws may have caused discrepancies across results in previous studies independently of the validity of the MTC account or whether the latter may be genuinely flawed. Our evidence so far points to the latter possibility. In particular, our findings challenge two MTC assumptions, namely that coordination expressions have default 
meanings that are captured by truth conditions and that stationary trials are difficult to process for reasons unrelated to early comprehension (i.e., unrelated to meaning retrieval). The first assumption was already difficult to sustain in the light of results reported in previous coordination studies (e.g., Braine and Rumain 1981; Paris 1973; Sternberg 1979), which were unclear as to why individuals may sometimes adopt an inclusive default meaning for disjunctions and at other times an exclusive default meaning. Similarly, it is unclear how the MTC view can accommodate response patterns for TT conjunction trials in our study. In particular, response latencies were twice as high in stationary trials compared with moving trials, indicating considerable processing difficulty. Nevertheless, it is in these contexts that we observed default meaning override for TT conjunctions, to use terms agreeable with the MTC view. Hence participants' preference for invalidating conjunction descriptions in such contexts would reflect a basic tendency to override the single meaning available for TT conjunctions, which is untenable.

The results of our study also challenge another assumption of the MTC view that stimuli perceptual properties affect the late stages of online comprehension. Specifically, the response patterns we obtained for valid control conditions (requiring a 'yes' response), namely for TF and FT disjunctions, lend support to our hypothesis that stimuli perceptual properties directly affect meaning (i.e., simulation) retrieval. Recall that the MTC account predicts no effect of stimuli properties on validation rates other than random errors in these conditions. Nevertheless, validation scores were significantly lower in stationary than in moving trials, even though comprehenders would not be prompted to making a choice between logically valid alternatives neither in TF nor in FT conditions.

Instead, we propose that visual stimuli properties affect meaning retrieval, which is an early phase of comprehension. Put differently, conjunction and disjunction do not have default and derived meanings but evoke each a single meaning-oneobject and two-object perceptual simulations, respectivelywhich can be retrieved either partly or fully, depending on context and task requirements. Therefore, comprehenders who validate TT disjunction descriptions, for instance, are not thereby selecting the 'inclusive' meaning, but merely acknowledging that visual stimuli match the simulation evoked by disjunction. Similarly, comprehenders who reject TT disjunction descriptions are not selecting the 'exclusive' meaning, but merely acknowledging that visual stimuli do not match disjunction simulation. We further propose that, in difficult tasks or contexts (e.g., stimuli with poor visual resolution, multitasking), individuals act as optimistic assessors (some more than others-for an introduction to optimism bias, see Sharot 2012) who assume, without adequate evidence, that stimuli properties necessary for successful simulation retrieval only appear to be missing. From this perspective, inclusive and exclusive disjunction meanings (i.e., 'yes' and 'no' answers) reflect the degree to which comprehenders are able to retrieve coordination simulations based on stimuli perceptual properties as well as on their willingness to accommodate or fill in missing information.

This brings us to the last point we wish to focus on, relating to individual differences in response patterns. For TT disjunction trials, for instance, $22 \%$ of participants consistently failed to validate them, while about $6 \%$ validated them across the board, suggesting that not all individuals were sensitive to grouping cues or that they were not using them for meaning retrieval. Indeed, findings from visual-search studies suggest that individuals differ in their ability to process visual information (e.g., Treisman and Gelade 1980, experiment VII). Further, elements defined by their color may be searched preferentially in relation to stimuli defined along other dimensions (e.g., Hannus et al. 2006; Poisson and Wilkinson 1992; Zohary and Hochstein 1989). For example, Williams (1966) investigated eye fixations involved in a search task in which stimuli were defined by a conjunction of size, color, or shape. He showed that, even when prompted to search for a particularly colored shape, participants still fixated more readily on stimuli defined by their color.

\section{Conclusion}

We determined that stimuli properties directly influence coordination validation rates in a study using concurrent visual and linguistic information. We thereby provided support for an embodied cognition account of coordination featuring object-like simulations. Further research will determine whether similar results obtain when stimuli belong to other perceptual modalities, either combined or in isolation, as when people listen to spoken language without looking at corresponding visual displays, for instance. It also remains to be determined whether other cues to object identification besides dependent or independent motion may help retrieve coordination simulations and how sparse these simulations could be in order to be retrieved safely. We may find either that simulations evoked by coordinators (and other abstract concepts) are tied to a specific modality or that these simulations can be easily represented in various modalities, in which case they must be inherently sparser than those evoked by concrete objects.

\section{References}

Barsalou LW (1999) Perceptual symbol systems. Behav Brain Sci 22:577-660 
Barsalou LW, Santos A, Simmons WK, Wilson CD (2008) Language and simulation in conceptual processing. In: De Vega M, Glenberg AM, Graesser AC (eds) Symbols, embodiment, and meaning. Oxford University Press, Oxford

Braine M, Rumain B (1981) Children's comprehension of "or": evidence for a sequence of competencies. J Exp Child Psychol 31:46-70

Bundesen C (1990) A theory of visual attention. Psychol Rev 97:523-547

De Neys W, Schaeken W (2007) When people are more logical under cognitive load: dual task impact on scalar implicature. Exp Psychol 54:128-133

Dumitru ML, Joergensen GH, Cruickshank AG, Altmann GTM (2013) Language-guided visual processing affects reasoning: the role of referential and spatial anchoring. Conscious Cogn 22(2):562-571

Duncan J, Humphreys GW (1989) Visual search and stimulus similarity. Psychol Rev 96:433-458

Egly R, Driver J, Rafal RD (1994) Shifting visual attention between objects and locations: evidence from normal and parietal lesion subjects. J Exp Psychol Gen 123:161-176

Fillenbaum S (1974) Or: some uses. J Exp Psychol 103:913-921

Glenberg AM (2007) Language and action: Creating sensible combinations of ideas. In: Gaskell G (ed) Oxford handbook of psycholinguistics. Oxford University Press, Oxford, pp 361-370

Hannus A, van den Berg R, Bekkering H, Roerdink JBTM, Cornelissen FW (2006) Visual search near threshold: some features are more equal than others. J Vision 6:525-540

Hauk O, Johnsrude I, Pulvermüller F (2004) Somatotopic representation of action words in human motor and premotor cortex. Neuron 41:301-307

Horn L (1972) On the semantic properties of the logical operators in English. PhD thesis, University of California at Los Angeles

Jiang Y, Olson IR, Chun M (2000) Organization of visual short-term memory. J Exp Psychol Learn Mem Cogn 26:683-702

Johnson J, Pashler H (1990) Close binding of identity and location in visual feature perception. J Exp Psychol Hum Percept Perform 16:843-856

Keenan EL, Faltz LM (1985) Boolean semantics for natural language. Reidel, Dordrecht

Lamy D, Egeth H (2002) Object-based selection: the role of attentional shifts. Percept Psychophys 64:52-66

Link G (1983) The logical analysis of plurals and mass terms: a lattice-theoretical approach. In: Baeuerle R et al (eds) Meaning, use, and interpretation of language. de Gruyter, Berlin, pp 302-323

Lupyan G (2008) From chair to "chair": a representational shift account of object labelling effects on memory. J Exp Psychol Gen 137:348-369
Meteyard L, Bahrami B, Vigliocco G (2007) Motion detection and motion verbs: language affects low-level visual perception. Psychol Sci 18:1007-1013

Nakayama K, He ZJ, Shimojo S (1995) Visual surface representation: a critical link between lower-level and higher-level vision. In: Kosslyn SM, Osherson DN (eds) Visual cognition, 2nd edn. MIT Press, Cambridge, pp 1-70

Nissen MJ (1985) Accessing features and objects: Is location special? In: Posner M, Marin O (eds) Attention and performance XI. Erlbaum, Hillsdale, pp 205-220

Paris S (1973) Comprehension of language connectives and propositional logical relationships. J Exp Child Psychol 16:278-291

Poisson ME, Wilkinson F (1992) Distractor ratio and grouping processes in visual conjunction search. Perception 21:21-38

Quinlan PT (1998) The recovery of identity and relative position from visual input: further evidence for the independence of processing of what and where. Percept Psychophys 60:303-318

Richardson DC, Spivey MJ, Barsalou LW, McRae K (2003) Spatial representations activated during real-time comprehension of verbs. Cogn Sci 27:767-780

Sagi D, Julesz B (1985) Where and what in vision. Science 228:1217-1219

Sharot T (2012) The optimism bias: why we're wired to look on the bright side. Constable \& Robinson Ltd, London

Simons DJ (1996) In sight, out of mind: when object representations fail. Psychol Sci 7:301-305

Simons M (2001) Disjunction and alternativeness. Linguist Philos 24:597-619

Sperber D, Wilson D (1986/1995) Relevance: communication and cognition. Blackwell, Oxford

Sternberg RJ (1979) Developmental patterns in the encoding and combination of logical connectives. J Exp Child Psychol 28:469-498

Tettamanti M, Buccino G, Saccuman MC, Gallese V, Danna M, Scifo $P$ et al (2005) Listening to action-related sentences activates fronto-parietal motor circuits. J Cogn Neurosci 17:273-281

Treisman AM, Gelade G (1980) A feature-integration theory of attention. Cogn Psychol 12:97-136

Treisman A, Sato S (1990) Conjunction search revisited. J Exp Psychol Hum Percept Perform 16:459-478

Williams LG (1966) The effect of target specification on objects fixated during visual search. Percept Psychophys 1:315-318

Zohary E, Hochstein S (1989) How serial is serial processing in vision? Perception 18:191-200

Zwaan RA, Stanfield RA, Yaxley RH (2002) Language comprehenders mentally represent the shapes of objects. Psychol Sci 13(2):168-171 